\title{
High SARS-CoV-2 Seroprevalence in Healthcare Workers in Bukavu, Eastern Democratic Republic of Congo
}

\author{
Denis Mukwege, ${ }^{1,2 *}$ Aline Kusinza Byabene,${ }^{2}$ Eric Mungu Akonkwa, ${ }^{1,2}$ Hafid Dahma, ${ }^{3}$ Nicolas Dauby, ${ }^{4,5,6}$ \\ Jean-Paul Cikwanine Buhendwa, ${ }^{2}$ Anne Le Coadou, ${ }^{5}$ Isabel Montesinos, ${ }^{3}$ Marie Bruyneel, ${ }^{7}$ Guy-Bernard Cadière, ${ }^{8}$ \\ Olivier Vandenberg, ${ }^{5,9}+$ and Yves Van Laethem ${ }^{4} \dagger$
}

${ }^{1}$ Gynaecology and General Surgery, Panzi General Referral Hospital, Bukavu, Democratic Republic of Congo; ${ }^{2}$ Faculty of Medicine, Evangelical University in Africa, Bukavu, Democratic Republic of Congo; ${ }^{3}$ Department of Microbiology, LHUB-ULB, Université Libre de Bruxelles, Brussels, Belgium; ${ }^{4}$ Department of Infectious Diseases, CHU Saint-Pierre, Université Libre de Bruxelles (ULB), Brussels, Belgium; ${ }^{5}$ Centre for Environmental Health and Occupational Health, School of Public Health, Université Libre de Bruxelles (ULB), Brussels, Belgium; ${ }^{\circ}$ Institute for Medical Immunology, Université Libre de Bruxelles (ULB), Brussels, Belgium; ${ }^{7}$ Department of Pulmonary Medicine, CHU Saint-Pierre, Université Libre de Bruxelles (ULB), Brussels, Belgium; ${ }^{8}$ Department of Gastrointestinal Surgery, European School of Laparoscopic Surgery, Saint-Pierre University Hospital, Université Libre de Bruxelles, Brussels, Belgium; ${ }^{9}$ Division of Infection and Immunity, Faculty of Medical Sciences, University College London, London, United Kingdom

\begin{abstract}
Among 359 healthcare workers (HCW) employed in Panzi General Referral Hospital located in Bukavu in the Democratic Republic of Congo, 148 (41.2\%) tested positive for SARS-CoV-2 antibodies. Thirty-three (22.3\%) of the 148 personnel with positive serology reported symptoms evoking a prior COVID-19 illness. None of the infected HCWs reported COVID-related hospitalization, and all of them recovered. Our findings indicate high and underestimated circulation of SARS-CoV-2 within the Bukavu area.
\end{abstract}

\section{INTRODUCTION}

The SARS-CoV-2 pandemic (causing COVID-19) hit Africa on February 25, 2020 and the Democratic Republic of the Congo (DRC) on March 10, 2020. ${ }^{1}$ At the time of this writing (December 27, 2020), a total of 16,038 confirmed COVID-19 cases with 566 deaths were reported in the DRC by the national authorities. Until now, there are only 394 SARS-CoV-2 infection-confirmed cases in the province of South-Kivu located at the eastern part of the DRC. However, because of limited testing capacity in the country, the total number of cases is likely largely underestimated.

Besides elderly people and patients with comorbidities, healthcare workers (HCWs) are considered as a high-risk population for SARS-CoV-2 infection. This is especially true in lowresource settings where personal protective equipment (PPE) and stringent infection prevention and control measures are lacking. Although many serological surveys have been performed in different industrialized countries assessing the risk for SARS-CoV-2 infection among frontline healthcare personnel, ${ }^{2,3}$ few studies have been published reporting serological testing in sub-Saharan countries. ${ }^{4}$ However, such studies are crucial for better organizing hospital response to the COVID-19 pandemic and also represent an opportunity to study natural infection in asymptomatic/pauci-symptomatic subjects and to estimate community transmission. ${ }^{4}$ The aim of the present work was to assess SARS-CoV-2 seroprevalence among frontline HCWs of the COVID-19 pandemic in Bukavu, the capital of the province of South-Kivu, and explore risk factors for seropositivity.

\section{MATERIALS AND METHODS}

Site of the study. The study was performed at the Panzi General Referral Hospital located in Bukavu, DRC. This

*Address correspondence to Denis Mukwege, Gynaecology and General Surgery, Panzi General Referral Hospital, Nobel Peace Prize laureate, Avenue de I'hopital 079, BP 266, Bukavu, Democratic Republic of Congo. E-mail: denismukwege@hotmail.com

$\dagger$ These authors contributed equally to this work. hospital of 350 beds is one of the main healthcare facilities of Bukavu, a city with more than 500,000 inhabitants. The hospital also serves as a reference center for the province of South-Kivu for the holistic care of survivors of sexual violence as well as maternal care and family planning. Since March 29, 2020, the date on which the first patient infected with SARSCoV-2 was declared in Bukavu, Panzi hospital has treated more than 218 patients suspected of COVID-19 infection, of which 121 have been confirmed by laboratory methods.

From July 2, 2020 to August 19, 2020, all staff members $(n=$ 393) working in Panzi General Referral Hospital located in Bukavu, DRC, were invited to participate in a seroprevalence study on a voluntary basis. Participants were asked to fill in a questionnaire with medical history and recent or current symptoms.

According to WHO guidelines (WHO/2019-nCoV/Surveillance Case_Definition/2020.1), suspected COVID-19 cases were defined by having an acute onset of any three or more of the following signs or symptoms in the previous 5 days: fever, cough, general weakness/fatigue, headache, myalgia, sore throat, coryza, dyspnea, anorexia/nausea/vomiting, diarrhea, and altered mental status. A probable COVID-19 case was defined as a person who meets the aforementioned clinical criteria and is a contact of a confirmed case or a person with onset of anosmia or ageusia in the absence of any other identified cause. A person with laboratory confirmation of COVID-19 infection, irrespective of clinical signs and symptoms, was considered as a confirmed COVID-19 case.

According provincial guidelines, diagnosis of SARS-CoV-2 infection was made on clinically suspected patients (including HCWs) on the nasopharyngeal swab sample by the use of COVID-19 antigen detection rapid diagnostic tests (RDTs) (Coris BioConcept, Gembloux, Belgium) and/or RT-PCR depending on test availability.

In the time frame of our study, first-line serological analysis was performed using a QuickZen COVID-19 IgM/lgG Kit (QuickZen) (ZenTech, Angleur, Belgium), a rapid point-of-care lateral flow immunoassays intended for the qualitative detection of IgG and IgM against SARS-CoV-2. Its combined IgM 
or IgG sensitivity and specificity were $71.1 \%$ and $100.0 \%$, respectively. ${ }^{7}$ All results were confirmed by Euroimmun AntiSARS-CoV-2 ELISA IgG assay (Euroimmun, Luebeck, Germany) showing a sensitivity and specificity of $61.7 \%$ and $98.6 \%$, respectively. $^{7}$

Descriptive statistics analysis was used to summarize the characteristics of our population, and the Fisher exact test and logistic regression for our categorical variables. All our $P$ values were two-tailed and considered statistically significant if $<0.05$. We used STATA 16.0 statistical software (version 9.4, StataCorp., College Station, TX).

Ethical approval for the study was granted by the provincial office of the National Committee of Health Ethics (CNES 001/ DPSK/153 PM/2020) regarding its compliance with the Guidelines for the Ethical Evaluation of Research Involving Human Subjects in the Democratic Republic of Congo (GEthicalEval - French) (2011). In the frame of this study, no recommendation for a written informed consent has been issued by the ethical committee considering that an informed oral consent obtained from each participant was sufficient. To ensure confidentiality, samples were analyzed anonymously.

\section{RESULTS}

A total of 359 HCWs $(91.4 \%$ of the 393 staff members working in Panzi General Referral Hospital), including physicians $(53,14.8 \%$ of the tested subjects), nurses $(83,23.1 \%)$, laboratory staff $(12,3.3 \%)$, paramedical staff $(101,28.1 \%)$, administrative staff $(36,10.0 \%)$, stretcher-bearers, and cleaners $(74,20.6 \%)$, agreed to participate in the study. Among them, 50.7\% (182/359) were male. Most were middleaged adults (mean age $=43.1$ years, $S D=11.3$ ) without chronic medical illnesses $(93.9 \%[n=337]$ reported no comorbidities). According to WHO criteria, 44 (12.3\%) HCWs reported symptoms evoking COVID-19 at the moment of sampling, and two (4.5\%) and $19(43.2 \%)$ of them were classified as suspected and probable COVID-19 cases, respectively, whereas 23 (52.3\%) had their clinical diagnosis confirmed by laboratory methods and therefore classified as confirmed COVID-19 cases.

The prevalence of IgM and IgG positivity was $24.0 \%$ (86/ $359)$ and $18.7 \%(67 / 359)$, respectively, when using the antibody (Ab) RDT as a first-line serological test only. When tested by using SARS-CoV-2 Euroimmun IgG ELISA assay, 148 $(41.2 \%)$ samples were positive for IgG. Among the 89 discordant IgG results between an Ab RDT and ELISA, 84 were retested with the Zentech RDT using a reader device. Among them, 56/84 and 19/84 were found positive for IgM and IgG, respectively. However, 39/56 HCWs with IgM only were also tested positive for IgG by SARS-CoV-2 Euroimmun IgG ELISA assay and therefore considered having specific IgM against SARS-CoV-2. A second serum was collected 3 months later from the 17 remaining HCWs, all of them showed a persistence of their lgM in the absence of any lgG. They were considered nonspecific IgM and seronegative for SARS-CoV-2 infection. However, because of the lack of reagents, we were unable to test for cross-reactivity or the presence of other infectious or inflammatory diseases. Overall, 148 (41.2\%) of the 359 participants had antibodies to SARS-CoV-2.

Only $33(22.3 \%)$ of the 148 personnel with positive serology reported symptoms evoking a prior COVID-19 illness. None of them suffered from severe infection. The characteristics of overall SARS-CoV-2 negative and SARS-CoV-2 positive population confirmed by serology are summarized in Table 1.

Even if our univariate analysis revealed a possible association between the SARS-CoV-2 infection and the gender, the type of work, or the presence of symptoms evoking COVID-19 at the moment of sampling, our multiple regression analysis showed that prior symptoms was the only risk factor significantly associated with the detection of SARS-CoV-2 antibodies $(P<0.001)$ (Table 2). Seropositivity appeared to be as common among those who reported not wearing PPE for all encounters versus those who reported always wearing PPE. No information was available on the adherence to the use of PPE by the HCWs. No impact of the reporting of a contact with a confirmed case of COVID-19 on the HCW's seropositivity was found.

\section{DISCUSSION}

In the start of SARS-CoV-2 outbreak in Africa, our main objective was to contain the spreading of the virus in the control strategy based on commitment and implementation of the diagnostic test in an integrative approach. ${ }^{6}$ However, such strategy was unsuccessful, and from July 2020, we observed a sharp increase in COVID-19-suspected patients attending Panzi hospital as reported in other regions of the DRC. ${ }^{1}$ Many challenges exist that compromise an efficient response toward the COVID-19 pandemic in the DRC. These hurdles include limited testing capacity, insufficient PPE and medical equipment, and limited technical capacity of COVID-19 case management and logistical resources in remote areas of the country. 6,8

We found a seroprevalence of IgG-specific SARS-CoV-2 of $41.2 \%$, which is much higher than that reported in a metaanalysis of seroprevalence (overall seroprevalence 7\% [95\% $\mathrm{Cl}: 4-11])$ in HCW in other parts of the world. ${ }^{3}$ However, a high seroprevalence level (31.6\%), which is higher than the general population, was also reported in a study involving 2,167 HCWs working in a London hospital. ${ }^{9}$ On the other hand, only limited seroprevalence studies have been performed so far in sub-Saharan Africa and in HCW especially. ${ }^{3}$ A study performed in urban Malawi that recruited 500 asymptomatic HCWs found a seroprevalence of $12.3 \% .^{4}$ Nevertheless, similar level as that we found in Panzi hospital was described in asymptomatic frontline $\mathrm{HCW}$ in Ibadan, Nigeria, where a seroprevalence of $45.1 \%$ was recently reported. ${ }^{10}$

Our findings suggest an early introduction of the virus in the Bukavu area that most probably went under-noticed. A modeling study indicates that characteristics of the African population, ${ }^{11}$ that is, an younger and more rural population might have impacted the dynamics of the epidemics as compared to other countries, resulting in widespread and mostly asymptomatic infections. ${ }^{12}$ In this cohort of middleaged (mean age: 43.1 years) and healthy $(93.9 \%$ without comorbidities) HCWs, SARS-CoV-2 infection was mild in the vast majority of cases. Accordingly, recent clinical experience with COVID-19 hospitalized patients in Kinshasa, DRC, ${ }^{13}$ indicates lower mortality than in industrialized countries. ${ }^{14}$

Among the different employees of Panzi hospital, we did not find any association between the type of work and the SARSCoV-2 seropositivity. This is in accordance with previous studies in Belgium and other countries, ${ }^{2,3,15}$ highlighting the efficacy of PPE when appropriately used. Previous experience 
TABLE 1

Characteristics of the overall, SARS-CoV-2 seronegative, and seropositive population

\begin{tabular}{|c|c|c|}
\hline & SARS-CoV-2 seronegative population $(n=211)$ & SARS-CoV-2 seropositive population $(n=148)$ \\
\hline Age (mean years $\pm S D$ ) & $43.4( \pm 11.5)$ & $42.7( \pm 11.1)$ \\
\hline \multicolumn{3}{|l|}{ Gender } \\
\hline Men & 119 (56.4\%) & $63(42.6 \%)$ \\
\hline Women & $92(43.6 \%)$ & $85(57.4 \%)$ \\
\hline \multicolumn{3}{|l|}{ Comorbidities } \\
\hline Cardiovascular history & $10(4.8 \%)$ & $3(2.0 \%)$ \\
\hline Respiratory pathology & $2(0.9 \%)$ & 0 \\
\hline Diabetes & $4(1.9 \%)$ & $1(0.7 \%)$ \\
\hline Obesity & $2(0.9 \%)$ & $4(2.7 \%)$ \\
\hline Kidney pathology & $1(0.5 \%)$ & 0 \\
\hline \multicolumn{3}{|l|}{ Equipment } \\
\hline FFP2 mask & $32(15.2 \%)$ & $26(17.6 \%)$ \\
\hline Surgical mask & $92(43.6 \%)$ & $73(49.3 \%)$ \\
\hline Cloth mask & $179(84.8 \%)$ & $126(85.1 \%)$ \\
\hline Gloves & $132(62.6 \%)$ & $91(61.5 \%)$ \\
\hline Disposable isolation gown & 49 (23.2\%) & 35 (23.6\%) \\
\hline Medical gown & $73(34.6 \%)$ & $66(44.6 \%)$ \\
\hline \multicolumn{3}{|l|}{ Contact with a confirmed case } \\
\hline Yes & $106(50.2 \%)$ & $89(60.1 \%)$ \\
\hline No & $105(49.8 \%)$ & 59 (39.9\%) \\
\hline \multicolumn{3}{|l|}{ Symptoms } \\
\hline Fever & $10(4.7 \%)$ & $26(17.6 \%)$ \\
\hline Shivers & $9(4.3 \%)$ & $24(16.2 \%)$ \\
\hline Chest pain & $10(4.7 \%)$ & $11(7.4 \%)$ \\
\hline Agesia/dysgesia & $1(0.5 \%)$ & $7(4.7 \%)$ \\
\hline Anosmia & $1(0.5 \%)$ & $10(6.8 \%)$ \\
\hline Dyspnea & $1(0.5 \%)$ & $1(0.7 \%)$ \\
\hline Conjunctivitis & $1(0.5 \%)$ & $1(0.7 \%)$ \\
\hline Skin rash & 0 & $2(1.3 \%)$ \\
\hline Headache & $24(11.4 \%)$ & $37(25.0 \%)$ \\
\hline Cough & $24(11.4 \%)$ & $26(17.6 \%)$ \\
\hline Asthenia & $10(4.8 \%)$ & $22(14.9 \%)$ \\
\hline Diarrhea & $8(3.8 \%)$ & $10(6.8 \%)$ \\
\hline Rhinorrhea & $28(13.3 \%)$ & $37(25.0 \%)$ \\
\hline Myalgia & $6(2.8 \%)$ & $22(14.9 \%)$ \\
\hline Altered mental status & $2(0.9 \%)$ & 0 \\
\hline No symptom & $156(73.9 \%)$ & $70(47.3 \%)$ \\
\hline \multicolumn{3}{|c|}{ Laboratory confirmation of COVID-19 infection } \\
\hline Positive & $1(0.5 \%)$ & $22(14.9 \%)$ \\
\hline Negative & $20(9.5 \%)$ & $15(10.1 \%)$ \\
\hline Not done & 190 (90.0\%) & $111(75.0 \%)$ \\
\hline \multicolumn{3}{|l|}{ COVID-19: case definitions } \\
\hline Suspect COVID-19 case $^{\star}$ & $1(0.5 \%)$ & $1(0.7 \%)$ \\
\hline Probable COVID-19 case $†$ & $9(4.3 \%)$ & $10(6.8 \%)$ \\
\hline Confirmed COVID-19 case & $1(0.5 \%)$ & $22(14.9 \%)$ \\
\hline No COVID-19 & $200(94.8 \%)$ & $115(77.8 \%)$ \\
\hline
\end{tabular}

of local HCWs in the DRC with recent Ebola outbreaks might have enhanced PPE correct use. ${ }^{16}$ This finding suggests that infection was probably related to community exposure. A study in Belgium found a strong association between household exposure and SARS-CoV-2 seropositivity $(\mathrm{OR}=$ 3.1). ${ }^{2}$ However, because no evaluation of PPE use was performed, this hypothesis should be confirmed by a seroprevalence study in the community but also phylogenetic studies comparing viral strains between hospital staff and COVID-19 patients. $^{17}$

As previously reported, self-reported symptoms were highly predictive of SARS-CoV-2 seropositivity. ${ }^{4}$ However, most of the seropositive HCWs did not report symptoms before sampling. Consequently, universal screening based on the combined use of RT-PCR and antigenic testing could improve the detection of asymptomatic HCWs and allow for appropriate infection control measure to limit the spread within healthcare institutions and into the community., ${ }^{44}$ In this time frame, to make regular screening more acceptable, alternative specimens (such as self-collected saliva) should be considered. ${ }^{18}$

As described previously, our study also underlines the limitation of the use of Ab RDT in low-resource settings. ${ }^{19}$ Even if their use allowed to rapidly detect some cases of infection, $54.7 \%(81 / 148)$ of the HCWs presenting IgG would have been missed if we would not have considered ELISA results. The failure of the Zentech RDT to correctly identify patients with SARS-CoV-2 IgM has already been described previously for other Ab RDTs and underlines the influence of nonspecific $\operatorname{lgM}{ }^{7}$ All of these points lead us to consider that field performance studies should be carried out in low- and middle-income countries to address the ability of RDTs to meet end-user's 
TABLE 2

Case-control study of demographic and clinical features of patients with SARS-CoV-2-negative and -positive IgG antibodies

\begin{tabular}{|c|c|c|c|c|c|c|}
\hline & $\begin{array}{c}\text { SARS-CoV-2 seronegative } \\
\text { population }(n=211)\end{array}$ & $\begin{array}{l}\text { SARS-CoV-2 seropositive } \\
\text { population }(n=148)\end{array}$ & Crude OR (95\% Cl) & $P$-value & Adjusted OR $(95 \% \mathrm{Cl}) \dagger$ & $P$-value $\dagger$ \\
\hline Age (years) & & & $0.9(1.09-1.0)$ & 0.58 & $1(0.9-1.0)$ & 0.87 \\
\hline Gender & & & & 0.01 & & 0.08 \\
\hline Men & $119(56.4 \%)$ & $63(42.6 \%)$ & $0.6(0.4-0.9)$ & & $0.6(0.4-1.0)$ & \\
\hline Women & $92(43.6 \%)$ & $85(57.4 \%)$ & 1 & & 1 & \\
\hline Comorbidities & & & & 0.63 & & 0.52 \\
\hline Yes & $14(6.6 \%)$ & $8(5.4 \%)$ & $0.8(0.3-2.0)$ & & $0.7(0.2-2.0)$ & \\
\hline No & $197(93.4 \%)$ & $140(94.6 \%)$ & 1 & & 1 & \\
\hline Type of work & & & & $0.05^{\star}$ & & $0.33^{*}$ \\
\hline Administrative staff & 19 (9.0\%) & $17(11.5 \%)$ & 1 & & 1 & \\
\hline Cleaning staff and stretcher-bearers & $55(26.1 \%)$ & $19(12.8 \%)$ & $0.4(0.2-0.9)$ & & $0.5(0.2-1.3)$ & \\
\hline Laboratory & $6(2.8 \%)$ & $6(4.0 \%)$ & $1.1(0.3-4.1)$ & & $1.0(0.2-4.3)$ & \\
\hline Paramedical staff & $58(27.5 \%)$ & $43(29.0 \%)$ & $0.8(0.4-1.8)$ & & $0.8(0.4-1.9)$ & \\
\hline Nurse & $41(19.4 \%)$ & $42(28.4 \%)$ & $1.1(0.5-2.5)$ & & $1.2(0.5-2.8)$ & \\
\hline Physician & $32(15.2 \%)$ & $21(14.2 \%)$ & $0.7(0.3-1.7)$ & & $0.7(0.3-1.8)$ & \\
\hline Use of personal protective equipment & & & & $0.69^{\star}$ & & $0.59^{*}$ \\
\hline Full & $71(33.6 \%)$ & $48(32.4 \%)$ & 1 & & 1 & \\
\hline Not full & $138(65.4 \%)$ & 97 (65.5\%) & $1.0(0.7-1.6)$ & & $1.3(0.7-2.2)$ & \\
\hline No & $2(0.9 \%)$ & $3(2.0 \%)$ & $2.2(0.3-13.8)$ & & $2.0(0.3-13.8)$ & \\
\hline Contact with a confirmed case & & & & 0.06 & & 0.69 \\
\hline Yes & $106(50.2 \%)$ & $89(60.1 \%)$ & $1.5(0.9-2.3)$ & & $1.1(0.7-1.9)$ & \\
\hline No & 105 (49.8\%) & 59 (39.9\%) & 1 & & 1 & \\
\hline WHO COVID-19 case definition & & & & $<0.001$ & & $<0.001$ \\
\hline Yes & $11(5.2 \%)$ & 33 (22.3\%) & $5.2(2.5-10.7)$ & & $5.1(2.3-11.0)$ & \\
\hline No & $200(94.8 \%)$ & $115(77.7 \%)$ & 1 & & 1 & \\
\hline
\end{tabular}

$\mathrm{OR}=$ odds ratio

${ }^{\star} P$-value by Wald test.

$\dagger$ Each $\mathrm{OR}$ and $P$-value are adjusted for all other variables in the (logistic regression).

expectation by fulfilling ASSURED criteria (affordable, sensitive, specific, user-friendly, rapid and robust, equipment-free, and deliverable to end-users) as recommended by the $\mathrm{WHO} .^{20}$

Our study has some limitations. The lack of reagents and disposables makes it difficult to assess the prevalence of the infection in the town of Bukavu and to compare data with other countries. In addition, we were not able to define whether infection was due to nosocomial (between hospital staff and/or through inanimate surfaces) or community transmission. A major strength is the concomitant testing serology by two different methods.

Our screening allowed us to show that all those working in Panzi hospital were highly exposed to SARS-COV-2 infection. No infected subjects developed severe symptoms requiring hospitalization, likely contributing to high and underestimated community transmission in the region.

Received November 30, 2020. Accepted for publication December 30, 2020.

Published online February 16, 2021.

Acknowledgments: We would like to thank all the hospital staff who worked hard during the pandemic and without whom this study would not have been possible. The American Society of Tropical Medicine and Hygiene has waived the Open Access fee for this article due to the ongoing COVID-19 pandemic.

Financial support: The study was funded partly by D. M. Foundation and ZenTech Biotechnology Company which provided the Rapid Diagnostic tests.

Disclosure: N. D. is a post-doctorate clinical master specialist of the F.R.S.-FNRS. This work is dedicated to the healthcare workers, the patients, and families affected by SARS-CoV-2.

Authors' addresses: Denis Mukwege, Department of Gynaecology and General Surgery, Panzi General Referral Hospital, Bukavu, Democratic Republic of Congo, and Faculty of Medicine, Evangelical University in Africa, Bukavu, Democratic Republic of Congo,
E-mail: denismukwege@hotmail.com. Aline Kusinza Byabene and Jean-Paul Cikwanine Buhendwa, Faculty of Medicine, Evangelical University in Africa, Bukavu, Democratic Republic of Congo, E-mails: alinekusinza@yahoo.fr and jpcikwa1@gmail.com. Eric Mungu Akonkwa, Department of Laboratory Medicine, Panzi General Referral Hospital, Bukavu, Democratic Republic of Congo, E-mail: ericmunguakonkwa2017@gmail.com. Hafid Dahma and Isabel Montesinos, Department of Microbiology, Laboratoire Hospitalir Universtaire de Bruxelles (LHUB-ULB), Brussels, Belgium, E-mails: hafid.dahma@lhub-ulb.be and mariaisabel.montesinoshernandez@ Ihub-ulb.be. Nicolas Dauby, Department of Infectious Diseases, Saint-Pierre University Hospital, Brussels, Belgium, E-mail: nicolas dauby@stpierre-bru.be. Anne Le Coadou, School of Public Health, Brussels, Belgium, E-mail: Anne.Le.Coadou@ulb.be. Marie Bruyneel, Department of Pulmonary Medicine, Saint-Pierre University Hospital, Brussels, Belgium, E-mail: marie.bruyneel@stpierre-bru.be. GuyBernard Cadière, Department of Gynaecology and General Surgery, Panzi General Referral Hospital, Bukavu, Democratic Republic of Congo, and Department of Gastrointestinal Surgery, European School of Laparoscopic Surgery, Saint-Pierre University Hospital, Brussels, Belgium, E-mail: cadieregb@gmail.com. Olivier Vandenberg, School of Public Health, Université Libre de Bruxelles, Brussels, Belgium, and Division of Infection and Immunity, Faculty of Medical Sciences, UCL, London, United Kingdom, E-mail: olivier.vandenberg@ulb.ac.be. Yves Van Laethem, Department of Infectious Diseases, Saint-Pierre University Hospital, Brussels, Belgium, E-mail: yves.vanlaethem@ stpierre-bru.be.

This is an open-access article distributed under the terms of the Creative Commons Attribution (CC-BY) License, which permits unrestricted use, distribution, and reproduction in any medium, provided the original author and source are credited.

\section{REFERENCES}

1. Juma CA, Mushabaa NK, Abdu Salam F, Ahmadi A, LuceroPrisno DE, III, 2020. COVID-19: the current situation in the Democratic Republic of Congo. Am J Trop Med Hyg 103: 2168-2170.

2. Steensels D, Oris E, Coninx L, Nuyens D, Delforge ML, Vermeersch P, Heylen L, 2020. Hospital-wide SARS-CoV-2 
antibody screening in 3056 staff in a tertiary center in Belgium. JAMA 324: 195-197.

3. Gómez-Ochoa SA, Franco OH, Rojas LZ, Raguindin PF, Roa-Díaz ZM, Wyssmann BM, Guevara SLR, Echeverría LE, Glisic M, Muka T, 2021. COVID-19 in health-care workers: a living systematic review and meta-analysis of prevalence, risk factors, clinical characteristics, and outcomes. Am J Epidemiol 190: 161-175.

4. Chibwana MG et al., 2020. High SARS-CoV-2 seroprevalence in health care workers but relatively low numbers of deaths in urban Malawi. medrxiv. doi: 10.1101/2020.07.30.20164970.

5. Mertens $P$ et al., 2020. Development and potential usefulness of the COVID-19 Ag respi-strip diagnostic assay in a pandemic context. Front Med 7: 225.

6. Mukwege D, Cadière GB, Vandenberg O, 2020. COVID-19 response in sub-saharan low-resource setting: healthcare soldiers need bullets. Am J Trop Med Hyg 103: 549-550.

7. Montesinos I et al., 2020. Evaluation of two automated and three rapid lateral flow immunoassays for the detection of anti-SARSCoV-2 antibodies. J Clin Virol 128: 104413.

8. Nachega JB et al., 2020. Responding to the challenge of the dual COVID-19 and Ebola epidemics in the Democratic Republic of Congo-priorities for achieving control. Am J Trop Med Hyg 103: 597-602.

9. Grant JJ, Wilmore SMS, McCann NS, Donnelly O, Lai RWL, Kinsella MJ, Rochford HL, Patel T, Kelsey MC, Andrews JA, 2020. Seroprevalence of SARS-CoV-2 antibodies in healthcare workers at a London NHS trust. Infect Control Hosp Epidemiol 1-3.

10. Olayanju O, Bamidele O, Edem F, Eseile B, Amoo A, Nwaokenye J, Udeh C, Oluwole G, Odok G, Awah N, 2021. SARS-CoV-2 seropositivity in asymptomatic frontline health workers in Ibadan, Nigeria. Am J Trop Med Hyg 104: 91-94.

11. Mbow M, Lell B, Jochems SP, Cisse B, Mboup S, Dewals BG, Jaye A, Dieye A, Yazdanbakhsh M, 2020. COVID-19 in Africa: dampening the storm? Science 369: 624-626.
12. Diop BZ, Ngom M, Pougué Biyong C, Pougué Biyong JN, 2020. The relatively young and rural population may limit the spread and severity of COVID-19 in Africa: a modelling study. BMJ Glob Health 5: e002699.

13. Nachega JB et al., 2020. Clinical characteristics and outcomes of patients hospitalized for COVID-19 in Africa: early insights from the Democratic Republic of the Congo. Am J Trop Med Hyg 369: 624-626.

14. Catteau L et al., 2020. Low-dose hydroxychloroquine therapy and mortality in hospitalised patients with COVID-19: a nationwide observational study of 8075 participants. Int J Antimicrob Agents 56: 106144.

15. Martin C, Montesinos I, Dauby N, Gilles C, Dahma H, Van Den Wijngaert S, De Wit S, Delforge M, Clumeck N, Vandenberg O, 2020. Dynamics of SARS-CoV-2 RT-PCR positivity and seroprevalence among high-risk healthcare workers and hospital staff. J Hosp Infect 106: 102-106.

16. Nachega JB, Mbala-Kingebeni P, Otshudiema J, Zumla A, TamFum JJM, 2020. The colliding epidemics of COVID-19, Ebola, and measles in the Democratic Republic of the Congo. Lancet Glob Health 8: e991-e992.

17. Houlihan CF et al., 2018. Use of whole-genome sequencing in the investigation of a nosocomial influenza virus outbreak. $J$ Infect Dis 218: 1485-1489.

18. Nagura-lkeda M et al., 2020. Clinical evaluation of self-collected saliva by quantitative reverse transcription-PCR (RT-QPCR), direct rt-qpcr, reverse transcription-loop-mediated isothermal amplification, and a rapid antigen test to diagnose COVID-19. J Clin Microbiol 58: e01438-20.

19. Jacobs J, Kühne V, Lunguya $O$, Affolabi D, Hardy L, Vandenberg O, 2020. Implementing COVID-19 (SARS-CoV-2) rapid diagnostic tests in sub-saharan Africa: a review. Front Med (Lausanne) 7: 557797.

20. Peeling RW, Holmes KK, Mabey D, Ronald A, 2006. Rapid tests for sexually transmitted infections (STIs): the way forward. Sex Transm Infect 82: v1-v6. 